Journal of Hard Tissue Biology 25[3] (2016) 282-287 (C) 2016 The Hard Tissue Biology Network Association Printed in Japan, All rights reserved. Original CODEN-JHTBFF, ISSN 1341-7649

\title{
Human Amniotic Membrane Extracts have Anti-Inflammatory Effects on Damaged Human Corneal Epithelial Cells In Vitro
}

\author{
Han-Na Lee ${ }^{1 *}$, Raymundo Bernardo ${ }^{1)^{*}}$, Gi-Yeon Han ${ }^{1)}$, Gyu-Young Kim ${ }^{1)}$, Ji-Soo Kim ${ }^{1)}$, Won-Yong Jung ${ }^{1)}$, \\ Keun-Nam Kim ${ }^{2)}$, Jae-Chan $\mathrm{Kim}^{3)}$ and Chan-Wha Kim ${ }^{1)}$ \\ 1) Department of Biotechnology, College of Life Sciences and Biotechnology, Korea University, Seoul, Korea \\ 2) Unimed Pharm. Inc. Seoul, Korea \\ 3) Department of Ophthalmology, Chung-Ang University Hospital, Seoul, Korea \\ (Accepted for publication, April, 15, 2016)
}

\begin{abstract}
The human amniotic membrane has therapeutic potential for several diseases such as cardiac ischemia, liver fibrosis, and ocular surface disorders. In the treatment of ocular surface disorders, human amniotic membrane transplantation promotes epithelial wound healing and suppresses inflammation. The objective of this study was to determine whether human amniotic membrane extracts (HAE) help damaged corneal epithelial cells recover from an inflammatory response. Human corneal epithelial cells (hCEC) which were induced inflammation were treated with human amniotic membrane extracts, and then the levels of inflammatory cytokines were measured. Human amniotic membrane extracts had an anti-inflammatory effect on damaged human corneal epithelial cells. More importantly, homogenized human amniotic membrane extracts of less than $3 \mathrm{kDa}$ had a greater capacity for reducing inflammation and secretion of interleukin-6 (IL-6) and interleukin-8 (IL-8). Thus, these results indicate that the use of human amniotic membrane extracts is a promising treatment for ocular surface disorders accompanied by inflammation.
\end{abstract}

Key words: Human amniotic membrane extract (HAE), Human corneal epithelial cell (hCEC), Anti-inflammatory effect, Interleukin-6 (IL-6), Interleukin-8 (IL-8)

\section{Introduction}

Dry eye syndrome (DES), also known as keratoconjunctivitis sicca (KCS), is a common disorder associated with increased osmolality of the tear film. DES can potentially damage to the ocular surface and results in various symptoms like ocular irritation, mucoid discharge, and excessive tearing with multifactor causes. DES also leads to an increase the risk of secondary infections ${ }^{1)}$. Research has demonstrated that inflammatory cytokines including interleukin-1 (IL-1), interleukin-6 (IL-6), interleukin-8 (IL-8), and tumor necrosis factor-alpha (TNF- $\alpha$ ) are highly expressed in tears of patients with multi-factorial DES ${ }^{2-7)}$. Moreover, current research suggests that neurogenic inflammation induced by neuropeptides may be closely associated with both the initial immune response and chronic inflammation in the disease $^{8}$. Thus, suppression of ocular inflammation and enhancement of tear film stability may be a therapeutic option in the treatment of DES.

The amnion is a membrane that protects the embryo during development. The human amniotic membrane has long been used

\footnotetext{
*These two authors contributed equally to the examination.

Correspondence to: Dr. Chan-Wha Kim, Department of Biotechnology, College of Life Sciences and Biotechnology, Korea University, 145, Anam-ro, Seongbuk-gu, Seoul 136-701, Korea; Tel: 82-2-3290-3439; Fax: 82-2-3290-3957; E-mail: cwkim@korea.ac.kr
}

clinically as biological dressings to heal skin wounds ${ }^{9)}$. Since the 1940s, human amniotic membranes have been applied as grafts or as patches for the management of ocular surface disorders ${ }^{10)}$. Human amniotic membrane extracts (HAEs) consist of a variety of molecules such as sugars, lipids, proteins, and peptides ${ }^{11)}$. Many studies have reported that the function of the amniotic membrane is known to exert anti-inflammation and anti-scarring effects and promote epithelial wound healing ${ }^{11-13)}$. Also, amniotic epithelial cells are reported to secrete immunosuppressive factors in previous studies. These studies showed that ocular surface inflammation was inhibited by the amniotic membrane ${ }^{14-18)}$. Despite the beneficial effects of amniotic membranes, their current clinical application requires transplantation, which is an invasive procedure.

In this study, we evaluated whether a suspension of amniotic membranes could have therapeutic effects. Human amniotic membranes were extracted with a homogenizer. We then tested whether the extract had an anti-inflammatory effect on damaged human corneal epithelial cells (hCECs). Finally, we investigated the anti-inflammatory effect of small molecule components of HAEs, which were isolated using a $3 \mathrm{kDa}$ cut-off centrifugal filter.

\section{Materials and Methods}

\section{Cell culture}


Han-Na Lee et al.: HAEs as Anti-Inflammatory Effects on Damaged hCECs

The human corneal epithelial cell (hCEC) line, CRL-11135 (ATCC, VA, USA), was cultured in Dulbecco's Modified Eagle Medium: Nutrient Mixture F-12 (DMEM/F12) (Invitrogen, CA, USA) supplemented with $10 \%$ heat-inactivated fetal bovine serum (FBS) and $100 \mathrm{U} / \mathrm{ml}$ penicillin-streptomycin at $37^{\circ} \mathrm{C}$ in an atmosphere containing $5 \% \mathrm{CO}_{2}$. The cell culture plates were precoated with a mixture of $0.01 \mathrm{mg} / \mathrm{mL}$ fibronectin (Sigma. Louis, MO, USA), $0.03 \mathrm{mg} / \mathrm{mL}$ bovine collagen type I (Sigma), and 0.01 $\mathrm{mg} / \mathrm{ml}$ bovine serum albumin (BSA) (Sigma). The medium was changed every 3 days.

\section{Ethics}

This study was approved by the Ethics Committee of ChungAng University Hospital. All procedures described here comply with Ethics Committee guidelines. The placenta with fetal membrane was obtained from Unimed Pharm. Inc. (Seoul, Korea). Informed consent was obtained from the mother, and the use of human placenta was approved by the clinics.

\section{Homogenization of human amniotic membranes}

The placenta was first cleaned with sterile phosphate-buffered saline (PBS) to remove blood clots and other contaminants. The separated amniotic membrane was thoroughly washed repeatedly with PBS supplemented with penicillin $(100 \mathrm{U} / \mathrm{ml})$ and streptomycin $(100 \mu \mathrm{g} / \mathrm{ml})$. The amniotic membrane was stored in chilled PBS containing a protease inhibitor cocktail (Sigma). The tissue was kept moist with chilled PBS throughout the entire procedure. The excised tissues were homogenized by homogenizer (Rose Scientific, Alberta, Canada) in chilled PBS containing a protease inhibitor cocktail. Homogenates were centrifuged at 3,000 rpm for $10 \mathrm{~min}$ and then at $12,000 \mathrm{rpm}$ for $20 \mathrm{~min}$ to obtain a clear supernatant. Extracts were immediately filtered through a $0.2 \mu \mathrm{m}$ filter (Sartorius-Stedim, Göttingen, Germany) to remove any microorganisms. Protein concentration was determined using the Bradford assay (Bio-Rad Laboratories, Hercules, CA, USA). The extract was then stored in aliquots at $-70^{\circ} \mathrm{C}$ until use.

\section{Separation of human amniotic membrane extracts (HAEs)}

HAEs were separated by size by using a Microcon centrifugal filter device (Millipore, Billerica, MA, USA), according to the manufacturer's protocol. Filtered HAEs were separated into 2 groups: those larger than $3 \mathrm{kDa}$ and those smaller than $3 \mathrm{kDa}$.

\section{Cell proliferation assay}

The cell proliferation assay was carried out using the Cell Counting Kit 8 (CCK-8) (Dojindo Laboratories, Kumamoto, Japan). Briefly, cells were cultured in 96 well culture plates. Cells were incubated with the homogenized HAEs for $72 \mathrm{hr}$ at $37^{\circ} \mathrm{C}$. After treatment, $10 \mu \mathrm{l}$ of the CCK-8 solution was added to each well, and the 96 well plates were incubated at $37^{\circ} \mathrm{C}$ for $2 \mathrm{hr}$. Optical densities (OD) of all wells were examined at a wavelength of 450 $\mathrm{nm}$ to determine cell viability.

\section{Preparation of chemically damaged hCECs and treatment with HAEs}

To generate chemically damaged hCECs, cells $\left(1 \times 10^{4}\right.$ cells/ $\mathrm{cm}^{2}$ ) were incubated with $200 \mu 1$ of $15 \%$ ethanol (EtOH) for 30 sec. The wells were washed, and the cells were cultured for $24 \mathrm{hr}$. The cells were then cultured for $24 \mathrm{hr}$ in conditioned media containing different concentrations of HAEs. After the treatment, the cells were washed with PBS and cultured for $24 \mathrm{hr}$.

\section{Caspase assay}

To measure caspase $3 / 7$ activity, hCECs were seeded in triplicate in a 96 well plate and incubated with $15 \%$ EtOH for 30 sec. After $72 \mathrm{hr}$, caspase 3/7 activity was assayed with a CaspaseGlo assay kit (Promega, Fitchburg, WI, USA), which includes a luminogenic caspase $3 / 7$ substrate, according to the manufacturer's protocol.

\section{Cytokine quantification}

Media from cultured hCECs were harvested, and IL-6 and IL-8 levels were measured using a commercially available ELISA kits (Koma Biotech, Seoul, Korea), according to the manufacturer's instructions.

\section{Statistical analysis}

Data were presented as the mean $\pm \mathrm{SD}$. Comparisons of continuous variables between more than two groups were performed by one way ANOVA, and t-tests were used to specify differences between groups. $\mathrm{P}<0.05$ was considered statistically significant. The SPSS software package was used for statistical tests (12.0.1).

\section{Results}

\section{Induction of inflammation in human corneal epithelial cells by using EtOH}

To generate the chemically-damaged hCECs, cells were incubated with $15 \% \mathrm{EtOH}$ for $30 \mathrm{sec}^{19)}$. To confirm the cell viability and inflammation effect of hCECs by $15 \% \mathrm{EtOH}$, viable cells were quantified by a CCK- 8 assay and the levels of IL- 6 and IL-8 were measured by ELISA. Morphology of the damaged cells was not changed (Fig. 1). However, as shown in Fig. 2a, cell viability decreased from $100 \%$ to $73 \%$ after exposure to EtOH. Chemical injury significantly increased IL-6 and IL-8 levels in the hCECs growth media (Fig. 2c, d) compared with the control group. In the cells treated with EtOH, IL-6 and IL-8 secretion increased from $100 \%$ to $121 \%$ and from $100 \%$ to $144 \%$, respectively.

When normal cells face environmental stress such as chemical 
a

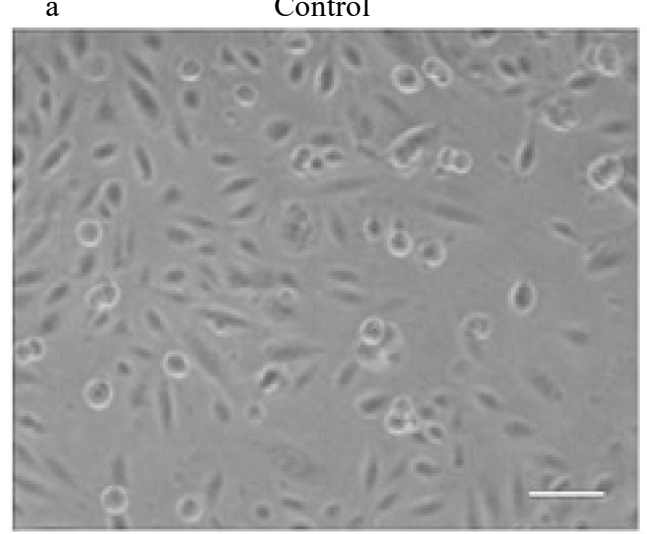

$\mathrm{b}$

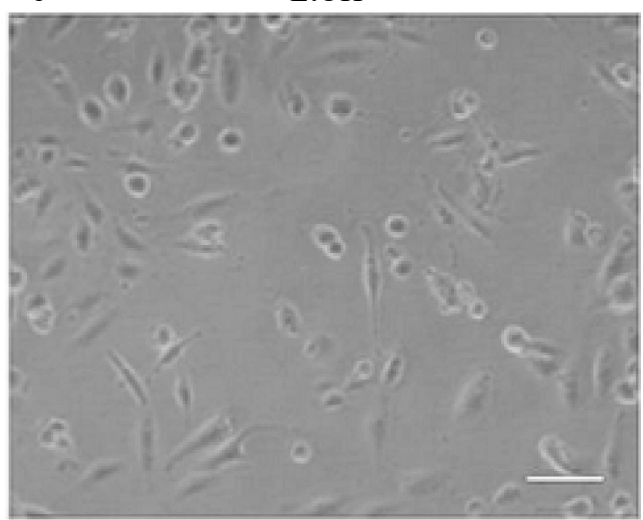

Figure 1. Cell morphology of hCECs (a) and chemically-damaged hCECs (b). (Scale bar $=100 \mu \mathrm{m})$.
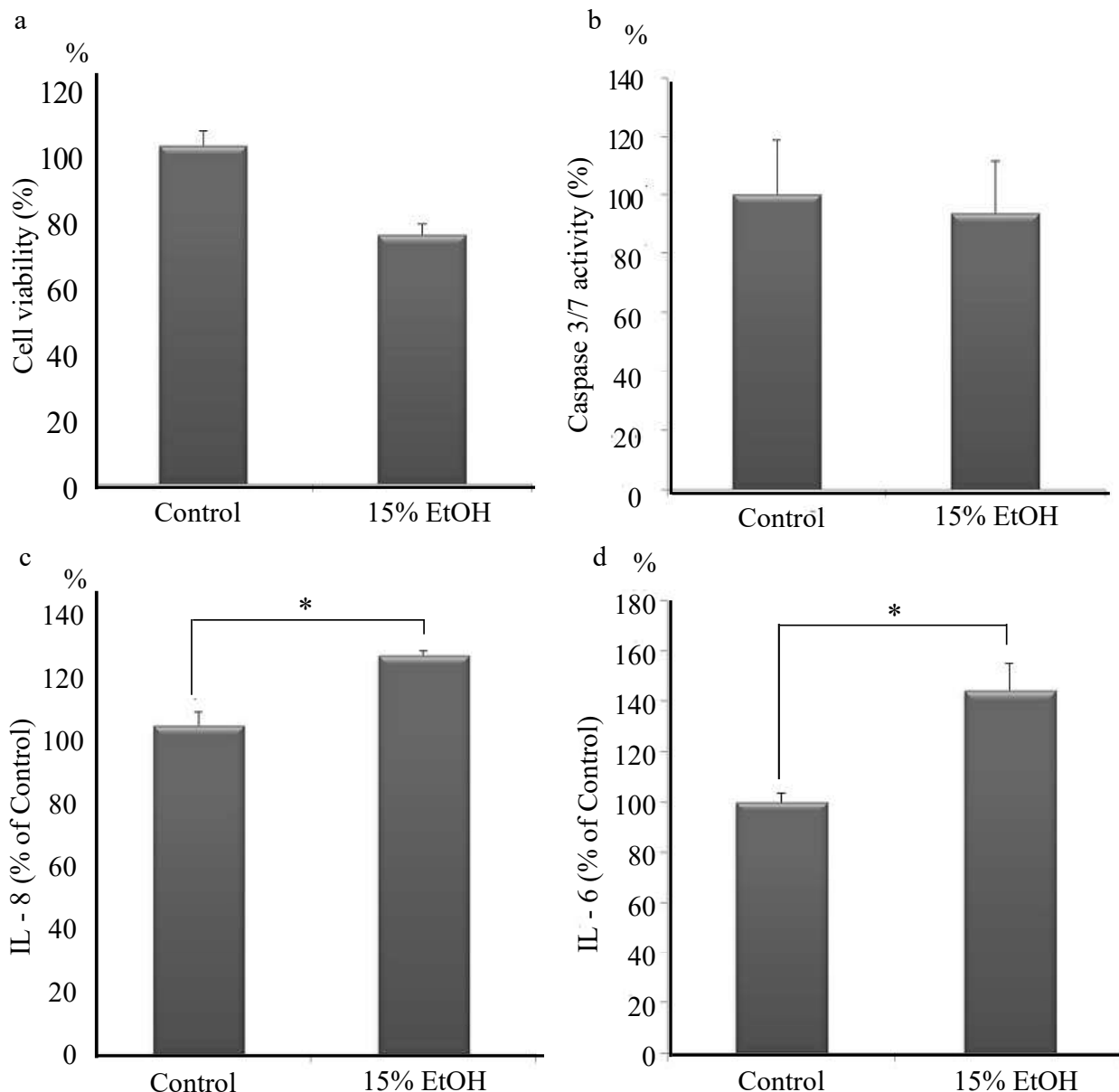

d $\%$

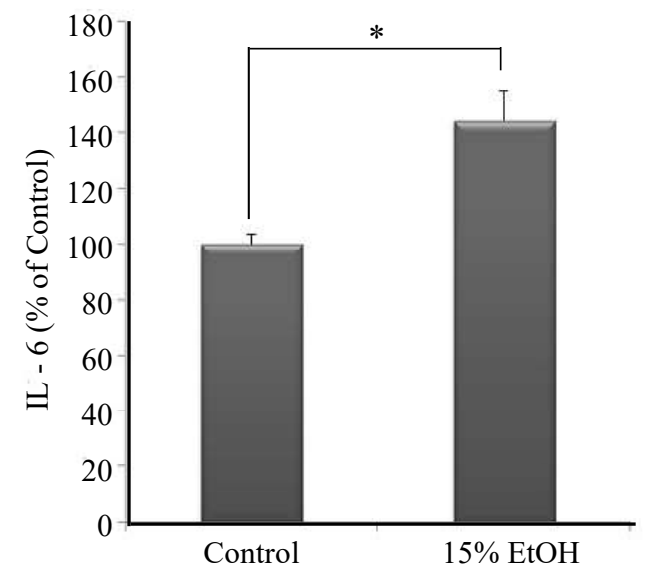

Figure 2. Inflammation is induced in the chemically injured human corneal epithelial cells (hCECs) by using $15 \%$ EtOH. (a) hCEC viability was measured by a cell proliferation assay. (b) Apoptosis in chemically damaged hCECs was determined by a caspase 3/7 assay. For the purpose of comparison, cell viability, levels of IL- 6 and IL-8, and caspase $3 / 7$ activity expressed by the control were considered to be $100 \%$. (c and d) The levels of IL- 6 and IL- 8 in hCECs culture media were detected by ELISA. Control group: non-treated hCECs, $15 \%$ EtOH group: hCECs treated with $15 \% \mathrm{EtOH}$ for $30 \mathrm{sec}$. Each bar represents the mean \pm SD of 3 independent experiments. Significant differences were determined using Student $t$-test $(* P<0.05)$.

exposure, high or low temperatures, and $\mathrm{pH}$ change, they undergo apoptosis. To determine whether our model of chemical injury had induced apoptosis, we measured the expression levels of caspase $3 / 7$, a reliable indicator of cellular apoptosis. As shown in Fig. 2b, caspase 3/7 levels did not change significantly (from $100 \%$ to $98 \%$ ) in hCECs treated with $\mathrm{EtOH}$, indicating that this chemical injury did not influence caspase $3 / 7$ related apoptosis. Taken together, our data demonstrate that $\mathrm{EtOH}$ treatment induced 
Han-Na Lee et al.: HAEs as Anti-Inflammatory Effects on Damaged hCECs

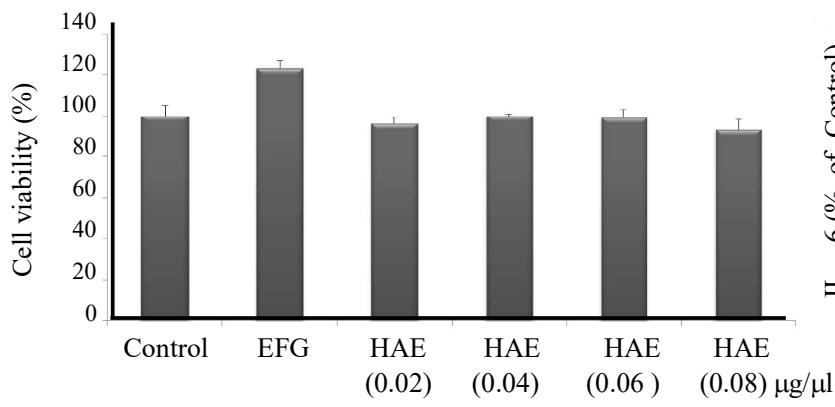

b

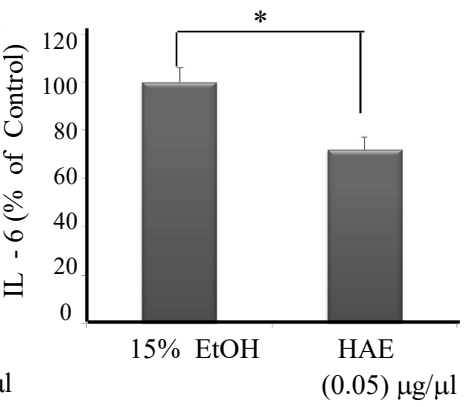

Figure 3. Human amniotic membrane extracts (HAEs) have an anti-inflammatory effect on hCECs. (a) hCECs were cultured in conditioned media containing $0.02,0.04,0.06$, or $0.08 \mu \mathrm{g} / \mathrm{ml}$ homogenized HAE. Epithelial growth factor was used as a positive control. For comparison, the cell viability expressed by the control (non-treated hCECs) was considered to be $100 \%$. (b) The EtOH-treated group was cultured in media for $24 \mathrm{hr}$ after chemical injury. The homogenized HAE-treated group was cultured in conditioned media containing homogenized HAE $(0.05 \mu \mathrm{g} / \mathrm{ml})$ for $24 \mathrm{hr}$ after chemical injury. The levels of IL-6 secretion were measured using ELISA. For the purpose of comparison, the amount expressed by the control (15\% EtOH-treated group) was considered to be $100 \%$. Each bar represents the mean \pm SD of 3 independent experiments. Significant differences were determined using Student $t$-test $(* P<0.05)$.

a

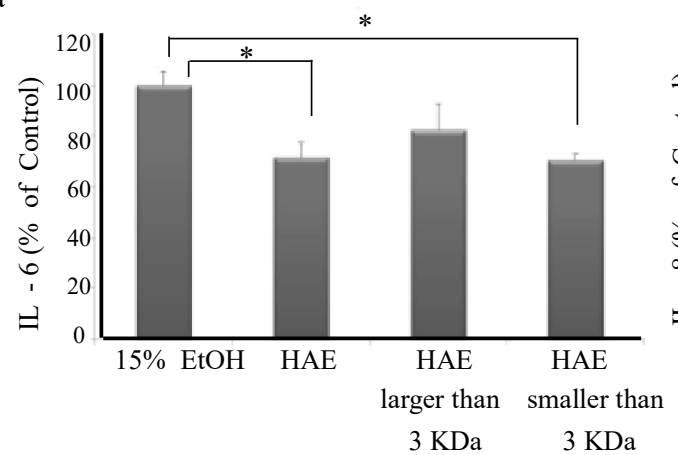

b

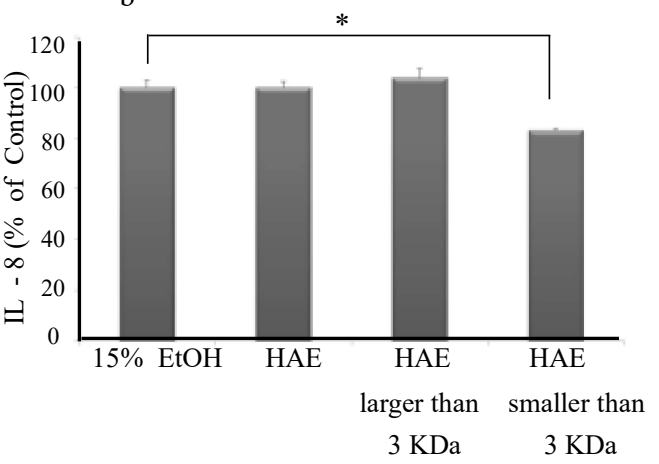

Figure 4. HAEs smaller than $3 \mathrm{kDa}$ have a greater anti-inflammatory effect than HAEs larger than $3 \mathrm{kDa}$. Chemically damaged hCECs were cultured in conditioned media containing homogenized HAEs $(0.05 \mu \mathrm{g} / \mathrm{ml})$, homogenized HAEs larger than $3 \mathrm{kDa}$, and homogenized HAEs smaller than $3 \mathrm{kDa}$ for $24 \mathrm{hr}$ after chemical injury. The levels of IL-6 (a) and IL-8 (b) secretion were measured using_ELISA. For the purpose of comparison, the amount expressed by each of the control (15\% EtOH-treated group) was considered to be $100 \%$. Each bar represents the mean \pm SD of 3 independent experiments. Significant differences were determined using one-way ANOVA $(* P<0.05)$.

inflammation without caspase-dependent apoptosis in hCECs.

\section{Human amniotic membrane extracts (HAEs) have an anti-} inflammatory effect on hCECs

We investigated whether HAEs have anti-inflammatory effects on chemically damaged hCECs. First, we examined whether HAEs are toxic to hCECs. hCECs were cultured with homogenized HAEs at 4 different concentrations $(0.02,0.04,0.06$ and $0.08 \mu \mathrm{g} / \mu \mathrm{l})$ for $72 \mathrm{hr}$. In this experiment, epithelial growth factor (EGF) was used as a positive control, and a solution containing no extracts was used as a negative control. As shown in Fig. 3a, HAEs have no significant effect on the proliferation rate of hCECs, and there is no variation in this proliferation rate according to $\mathrm{HAE}$ concentration. Thus, HAEs at $0.05 \mu \mathrm{g} / \mu \mathrm{l}$ were used in the next experiment.

Next, we directly examined the anti-inflammatory effects of homogenized HAEs. We treated hCECs, which had been previously treated with $15 \% \mathrm{EtOH}$ to induce inflammation, with homogenized HAEs $(0.05 \mu \mathrm{g} / \mu \mathrm{l})$ for $24 \mathrm{hr}$. As shown in Fig. 3b, homogenized HAEs significantly decreased IL-6 secretion from $100 \%$ to $71 \%$ in chemically injured hCECs. Therefore, we concluded that HAEs reduce inflammation in damaged hCECs.

\section{HAEs smaller than $3 \mathrm{kDa}$ have a greater anti-inflammatory effect than HAEs larger than $3 \mathrm{kDa}$}

To determine which fraction of homogenized HAEs contains anti-inflammatory factors, we used a Microconcentrifugal filter to separate the homogenized HAEs into 2 fractions: those larger than $3 \mathrm{kDa}$ and those smaller than $3 \mathrm{kDa}$. Chemically damaged hCECs were cultured with conditioned media containing homogenized HAEs, HAEs larger than $3 \mathrm{kDa}$, or HAEs smaller than $3 \mathrm{kDa}$. The IL-6 secretion from each group was then measured. As shown in Fig. 4a, IL-6 levels in HAE-treated groups were significantly decreased compared to those in the control $(15 \%$ EtOH treated group). In addition, we measured IL-8 secretion levels in the control (15\% EtOH treated group) and HAE-treated 
J.Hard Tissue Biology Vol. 25(3): 282 -287, 2016

groups. IL-8 secretion was slightly reduced in hCECs treated with whole HAEs and HAEs over $3 \mathrm{kDa}$. Interestingly, hCECs treated with HAEs smaller than $3 \mathrm{kDa}$ showed a significant decrease in IL-8 secretion, from $100 \%$ to $83 \%$ (Fig. 4b). These data indicate that HAEs smaller than $3 \mathrm{kDa}$ had a greater anti-inflammatory effect than whole HAEs or HAEs greater than $3 \mathrm{kDa}$. Therefore, we concluded that small molecules present in HAEs are important factors in reducing the inflammatory reaction of hCECs.

\section{Discussion}

The mechanisms of DES are related to tear hyperosmolality and tear film instability. Tear hyperosmolality, which arises from excessive water evaporation at the exposed ocular surface, leads to ocular surface inflammation with a cascade of inflammatory events. This result in tear film instability is considered as one of the symptoms in DES because it causes alterations of the tear film ${ }^{20)}$. Previous reports showed that tear concentrations of inflammatory cytokines IL-6 and IL-8 were significantly higher in dysfunctional tear syndrome. Detection of elevated IL-6 concentrations suggests an existence of inflammation induced disease because significant correlation was observed between IL6 and irritation symptoms ${ }^{21-22)}$. Therefore, treatments reducing inflammation of damaged corneal epithelia may be helpful in alleviating the symptoms of DES.

In the present study, we induced inflammation in hCECs in vitro with $\mathrm{EtOH}$. This chemical injury reduced cell viability greatly induces inflammation via significantly increasing IL-6 and IL-8 levels. Next, we investigated whether HAEs have an antiinflammatory effect on damaged hCECs and we discovered that the anti-inflammatory effect of HAEs $(0.05 \mu \mathrm{g} / \mu \mathrm{l})$ was superior to that of the negative control. Hence, this indicates that HAEs can alleviate inflammatory reactions. Finally, we attempted to identify which fraction of HAEs acts as an anti-inflammation mediator. HAEs smaller than $3 \mathrm{kDa}$ have an anti-inflammatory effect by predominantly suppressing IL- 6 and IL- 8 . We concluded that HAEs smaller than $3 \mathrm{kDa}$ are the most beneficial.

Recently, the use of amniotic membranes as a graft for ocular surface reconstruction has increased in popularity ${ }^{12)}$. Many studies have revealed amniotic membrane transplantation as a temporary or permanent graft that induces epithelial wound healing. It also possesses anti-inflammatory and anti-scarring effects on the ocular surface ${ }^{11,13)}$. Because of these beneficial effects, amniotic membranes are clinically used as a graft or a patch-in severe case of ocular surface disease. Furthermore, current research suggests that amniotic membrane suspensions aid corneal epithelial wound healing by promoting cell migration and proliferation ${ }^{23)}$. Thus, we hypothesized that amniotic membranes in a suspension form would also reduce the inflammatory reaction. As described in the Results section, HAEs exerted an anti-inflammatory effect on corneal epithelia without inducing apoptosis. This suggests that
HAEs may be a therapeutic option for epithelial defects accompanied by inflammation.

In this study, we demonstrated that HAEs smaller than $3 \mathrm{kDa}$, generated using a $3 \mathrm{kDa}$ molecular weight cut-off membrane, have greater anti-inflammatory activity than HAEs larger than $3 \mathrm{kDa}$. The HAE filtrate consists of diverse molecules, such as peptides, amino acids, and nucleotides. Previous studies have reported that peptides smaller than $3 \mathrm{kDa}$ in size possess antioxidant activities ${ }^{24-}$ 26).

The suspension form of amniotic membranes can have therapeutic effects on corneal epithelia, suppressing the inflammatory response. Specifically, HAEs smaller than $3 \mathrm{kDa}$ in size significantly contribute to its anti-inflammatory effect. Although further investigation is needed, these findings may provide a therapeutic approach for mild ocular surface disorders accompanied by inflammation. In particular, the use of small molecules may ultimately provide a new, targeted approach for the therapeutic treatment of mild ocular surface disorders accompanied by inflammation.

\section{Acknowledgements}

This study was supported by a grant from School of Life Sciences and Biotechnology for BK21 PLUS, Korea University. This work was also supported by Unimed Pharm. Inc. and ChungAng University Hospital.

\section{Conflict of Interest}

The authors have no COI existed.

\section{References}

1. Versura P, Profazio V, Cellini M, Torreggiani A and Caramazza R. Eye discomfort and air pollution. Ophthalmologica 213: 103-109, 1999

2. Erdogan-Poyraz C, Mocan MC, Bozkurt B, Gariboglu S, Irkec $M$ and Orhan M. Elevated tear interleukin-6 and interleukin-8 levels in patients with conjunctivochalasis. Cornea 28: 189-193, 2009

3. Tishler M, Yaron I, Geyer O, Shirazi I, Naftaliev E and Yaron M. Elevated tear interleukin-6 levels in patients with Sjogren syndrome. Ophthalmology 105: 2327-2329, 1998

4. Pflugfelder SC, Jones D, Ji Z, Afonso A and Monroy D. Altered cytokine balance in the tear fluid and conjunctiva of patients with Sjogren's syndrome keratoconjunctivitis sicca. Curr Eye Res 19: 201-211, 1999

5. Solomon A, Dursun D, Liu Z, Xie Y, Macri A and Pflugfelder SC. Pro- and anti-inflammatory forms of interleukin-1 in the tear fluid and conjunctiva of patients with dry-eye disease. Invest Ophthalmol Vis Sci 42: 2283-2292, 2001

6. Narayanan S, Miller WL and McDermott AM. Expression of human beta-defensins in conjunctival epithelium: 
Han-Na Lee et al.: HAEs as Anti-Inflammatory Effects on Damaged hCECs

relevance to dry eye disease. Invest Ophthalmol Vis Sci 44: 3795-3801, 2003

7. Yoon KC, Jeong IY, Park YG and Yang SY. Interleukin-6 and tumor necrosis factor-alpha levels in tears of patients with dry eye syndrome. Cornea 26: 431-437, 2007

8. Mantelli F, Micera A, Sacchetti M and Bonini S. Neurogenic inflammation of the ocular surface. Curr Opin Allergy Clin Immunol 10: 498-504, 2010

9. Hanada K, Shimazaki J, Shimmura S and Tsubota K. Multilayered amniotic membrane transplantation for severe ulceration of the cornea and sclera. Am J Ophthalmol 131: 324-331, 2001

10. Toda A, Okabe M, Yoshida T and Nikaido T. The potential of amniotic membrane/amnion-derived cells for regeneration of various tissues. J Pharmacol Sci 105: 215-228, 2007.

11. Tseng SC, Espana EM, Kawakita T, Di Pascuale MA, Li W, He H, Liu TS, Cho TH, Gao YY, Yeh LK and Liu CY. How does amniotic membrane work? Ocul Surf 2: 177-187, 2004

12. Kim JC and Tseng SC. Transplantation of preserved human amniotic membrane for surface reconstruction in severely damaged rabbit corneas. Cornea 14: 473-484, 1995

13. Rauz S and Saw VP. Serum eye drops, amniotic membrane and limbal epithelial stem cells - tools in the treatment of ocular surface disease. Cell Tissue Bank 11: 13-27, 2010

14. Azuara-Blanco A, Pillai CT and Dua HS. Amniotic membrane transplantation for ocular surface reconstruction. Br J Ophthalmol 83: 399-402, 1999

15. Chen HJ, Pires RT and Tseng SC. Amniotic membrane transplantation for severe neurotrophic corneal ulcers. Br J Ophthalmol 84: 826-833, 2000.

16. Kim JS, Kim JC, Hahn TW and Park WC. Amniotic membrane transplantation in infectious corneal ulcer. Cornea 20: 720726, 2001

17. Kim JS, Kim JC, Na BK, Jeong JM and Song CY. Amniotic membrane patching promotes healing and inhibits proteinase activity on wound healing following acute corneal alkali burn. Exp Eye Res 70: 329-337, 2000

18. Li H, Niederkorn JY, Neelam S, Mayhew E, Word RA, McCulley JP and Alizadeh H. Immunosuppressive factors secreted by human amniotic epithelial cells. Invest Ophthalmol Vis Sci 46: 900-907, 2005.

19. Oh JY, Kim MK, Shin MS, Wee WR and Lee JH. Cytokine secretion by human mesenchymal stem cells cocultured with damaged corneal epithelial cells. Cytokine 46: 100-103, 2009

20. Stahl U, Willcox M and Stapleton F. Osmolality and tear film dynamics. Clin Exp Optom 95: 3-11, 2012

21. Lam H, Bleiden L, de Paiva CS, Farley W, Stern ME and Pflugfelder SC. Tear cytokine profiles in dysfunctional tear syndrome. Am J Ophthalmol 147: 198-205, 2009

22. Pflugfelder SC. Anti-inflammatory therapy of dry eye. Ocul Surf 1: 31-36, 2003

23. Choi JA, Jin HJ, Jung S, Yang E, Choi JS, Chung SH and Joo CK. Effects of amniotic membrane suspension in human corneal wound healing in vitro. Mol Vis 15: 2230-2238, 2009

24. Di Bernardini R, Rai DK, Bolton D, Kerry J, O’Neill E, Mullen AM, Harnedy P and Hayes M. Isolation, purification and characterization of antioxidant peptidic fractions from a bovine liver sarcoplasmic protein thermolysin hydrolyzate. Peptides 32: 388-400, 2011

25. Saiga A, Tanabe $\mathrm{S}$ and Nishimura T. Antioxidant activity of peptides obtained from porcine myofibrillar proteins by protease treatment. J Agric Food Chem 51: 3661-3667, 2003

26. Saito K, Jin DH, Ogawa T, Muramoto K, Hatakeyama E, Yasuhara T and Nokihara K. Antioxidative properties of tripeptide libraries prepared by the combinatorial chemistry. J Agric Food Chem 51: 3668-3674, 2003 